\title{
Design towards Higher Integration Levels in Power Electronics
}

\author{
E.C.W. de Jong \\ Delft University of Technology \\ Delft, The Netherlands \\ Email: E.C.W.deJong@EWI.TUDelft.nl
}

\author{
J. Popović \\ Delft University of Technology \\ Delft, The Netherlands \\ Email: J.Popovic@EWI.TUDelft.nl
}

\author{
J.A. Ferreira \\ Delft University of Technology \\ Delft, The Netherlands \\ Email: J.A.Ferreira@EWI.TUDelft.nl
}

\begin{abstract}
A giant leap forward for converters in the power electronic industry is possible by increasing the level of integration, i.e. by integrating the function performed by several discrete parts (electrical, thermal, mechanical, packaging, etc.) into one single integrated part. By incorporating these multifunctional parts and components, instead of conventional discrete component technology, a converter can be put together using fewer parts and less manufacturing processes, reducing size and cost. To comment on the current level of integration of any given converter requires that the, often subjective, implementation of the three issues: packaging, integration and thermal management be scrutinized. Figures of merit are introduced here to provide a systematic way to perform exactly that, in order to advance towards designing converters for higher integration levels. If these figures of merit are adopted in converter design one can systematically design power electronics with higher integration levels resulting in cheaper, more power dense converters enabling the power electronic industry to meet the low cost and high performance demand.
\end{abstract}

\section{INTRODUCTION}

The power electronic industry is under continual pressure for lower cost and higher performance in power converters. To keep on delivering converters to meet these demands require a continual improvement in not only electrical design, but also material- and manufacturing technology. A means to directly address these aspects is to increase the integration level of converters, i.e. by integrating the function performed by several discrete parts (electrical, thermal, mechanical, packaging, etc.) into one single integrated part [1]-[4]. This emerging method enables a reduction of the large number of discrete parts that have to be manufactured and assembled, and in doing so directly reduces their size and cost. Gradually more technologies addressing packaging, integration of passives and thermal management are being developed to achieve these higher integration levels, such as PCB and silicon integrated magnetics. [5]-[8]. However, during the design process it remains difficult to comment on the impact of a proposed conceptual design, as the choice of technology to use (mainly dependant on the vision and creativity of the responsible design engineer) has substantial influence on the resulting converter, its physical realisation, its performance as well as its impact on manufacturing. To be able to do this in a systematic way requires figures of merit. Concerning electrical circuit design, a good number of figures of merit are already used. Examples are electrical efficiency $(\eta)$, total harmonic distortion (THD) and the EMI spectra. For packaging, integration and thermal management such figures of merit do not exist yet. Therefore, figures of merit have been developed to quantify the level of integration in power electronic converters and effectiveness of thermal management. If these figures of merit are adopted in converter design one can systematically design power electronics with higher integration levels resulting in cheaper, more power dense converters enabling the power electronic industry to meet the low cost and high performance demand.

This publication outlines the developed figures of merit in Section II, and applies it during a volumetric optimisation of a power electronic converter in Section III.

\section{Performance indicAtors}

The integral aspects: integration and packaging, as well as thermal management are addressed after which the application on a conceptual converter is presented.

\section{A. Integration and packaging}

The large number of construction parts that have to be manufactured and assembled, makes the cost and size of converters large. To improve the construction of these converters, the way discrete component technology is being implemented has to be revised. To this extent, a power electronic converter is broken down to its basic constructional parts: functional elements parts that perform fundamental functions (electrical and thermal), such as power and IC dies as semiconductor functional elements, metallized film roll as capacitive functional element, wire or planar copper conductors and magnetic core as magnetic functional elements; and packaging elements - parts that perform packaging functions (electrical interconnection, insulation, mechanical support, heat removal and protection), such as semiconductors lead frames, components leads, cases, bobbin, PCB dielectric etc. Using construction parts in a multi-functional way, i.e. integration, the power electronic converter can be reassembled with fewer constructional parts and manufacturing processes. If a number of parts in a subcircuit are integrated in one part that performs the same function, fewer manufacturing processes and less material should be employed reducing both cost and size. Depending on the subject of integration, two types of integration can be distinguished: functional elements integration and packaging elements integration [9].

1) Functional elements integration level: Functional elements integration occurs when several functional elements are substituted with one that performs the same fundamental functions, for example: monolithic semiconductor integration, electromagnetic integration of passives, integration technologies such 


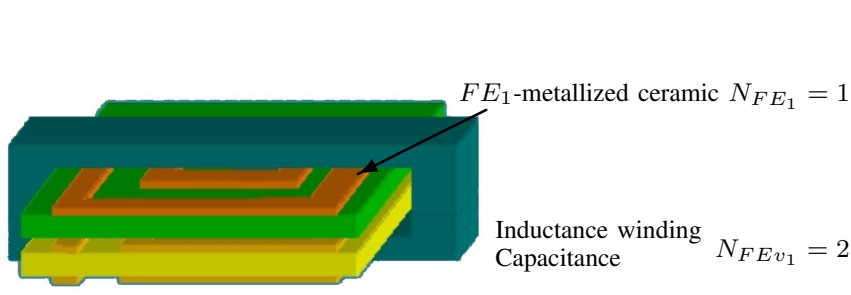

(a) functional elements integration - planar LC

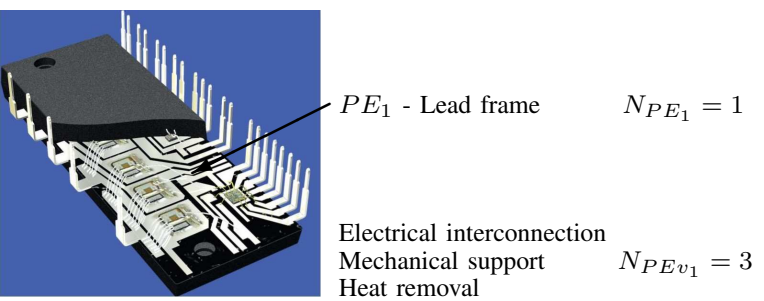

(b) packaging elements integration - lead frame power module [10]

Fig. 1. Integration level

as thick film, LTCC and PCB embedded passives. The main benefits of functional elements integration are reduction of the number of parts and manufacturing processes, fewer assembly steps, volume reduction, better performance due to lower parasitic values and a potential for improved reliability due to fewer interconnects. In order to evaluate the level of functional elements integration in a converter, a functional elements integration level quantity is introduced. This quantity shows how many electrical or thermal circuit symbols, functional elements in a sub-circuit perform on average. For this a virtual functional element needs to be defined: a functional element that contributes to $n$ circuit elements (circuit schematic symbols) in the discrete circuit schematic is "worth" $n$ virtual functional elements.

The quantity functional elements integration level $\left(K_{I}\right)$ is then defined as:

$$
K_{I}=\frac{\sum_{i} N_{F E v_{i}}}{N_{F E}}=\frac{N_{F E v}}{N_{F E}}
$$

where $N_{F E}$ is the total number of functional elements in the converter, $N_{F E v_{i}}$ is the number of virtual functional elements that the functional element $i$ is worth, and $N_{F E v}$ is the total number of virtual functional elements in the converter. As an example, copper tracks in a planar integrated LCT element represent one functional element. They take part in three circuit elements: inductance (windings), transformer (windings) and capacitance (electrodes), hence this element is worth three virtual functional elements (Figure 1(a)). The level of functional elements integration $K_{I}$ can be increased by means of several methods:

- Multifunctional functional elements (several circuit symbols implemented in one functional element). Typical examples are: monolithic semiconductor integration, electromagnetic integration etc.

- Integrated processes (implementing a number of circuit symbols in the same process). Typical examples are: hybrid integration technologies (thick film and thin film), PCB embedded passives etc.

- Packaging element as functional element (a packaging element is designed to implement a circuit symbol). An example of this integration method is using an electrical interconnection packaging element (copper tracks on $\mathrm{PCB})$ to implement magnetic windings.
2) Packaging elements integration level: Packaging elements integration occurs when several of packaging elements are substituted with one that performs the same packaging functions. It can be implemented by integrating a number of functional elements in one package, where they share the same packaging elements, such as the housing, mechanical support, thermal paths or when a packaging element is designed to be multifunctional, i.e. to perform electrical interconnection, mechanical support and heat removal function, for example: power multichip modules and chip-on-board (COB). The main benefits of packaging elements integration are reduction of the number of parts (packaging elements only since this type of integration generally does not influence functional elements), fewer manufacturing processes and assembly steps, improved performance due to fewer parasitics and volume reduction. Similarly to functional elements integration level, a quantity that evaluates level of packaging elements integration, packaging elements integration level $\left(K_{P}\right)$, is defined as

$$
K_{P}=\frac{\sum_{j} N_{P E v_{j}}}{N_{P E}}=\frac{N_{P E v}}{N_{P E}}
$$

where $N_{P E}$ is the total number of packaging elements while $N_{P E v}$ is the total number of virtual packaging elements in the sub-circuit. As an example, the lead frame in a power module represents one packaging element. Since it provides mechanical support, a heat path as well as electrical interconnection, it acts as three virtual packaging elements, i.e. $N_{P E v}$ of this element is 3 (Figure 1(b)). The level of packaging elements integration $K_{P}$ can be increased by means of several methods:

- Multifunctional packaging elements (several packaging functions performed by one packaging element). A typical example is a lead-frame based power module, where the lead-frame performs electrical interconnections, mechanical support and heat conduction)

- Sharing packaging elements (a number of functional elements share a packaging element). Multichip modules (MCM) where a number of discrete semiconductor dies share a common ceramic substrate are an example of this integration method.

- Reducing the number of packaging levels (transferring some of the packaging functions from the component level to the assembly level)

- Functional element as packaging element (functional el- 
ement performs one or more packaging functions).

\section{B. Thermal management}

The two thermal management performance indicators indicate the per volume heat which a converters thermal management is submitted to, as well as the thermal performance of the components together with their margin for improvement [11]. 1) Thermal management loss density (TMLD): quantifies the effective use of implemented material and parts that perform a thermal management function. It assesses the level of thermal loading of material and subsequently comments on the level of power density attainable in electronic assemblies. Similar to power density, the thermal management loss density relates power to volume, where $P_{\text {losses }}$ is the dissipated electrical loss (heat) and $V_{T M}$ the volume invested in thermal management [11]. It is defined as the ratio between the electrical losses that need to be removed from a system and the volume of the thermal management material that needs to transport the heat caused by these losses and is given by

$$
T M L D=\frac{P_{\text {losses }}}{V_{T M}}
$$

measured in $W / m^{3}$. $P_{\text {losses }}$ represent the total power dissipation. $V_{T M}$ represents the volume of all the material contributing towards the thermal management of the converter - silicon die in semiconductor devices; electrically conductive paths, these include material for wire bonds, electrical package pins and tabs; package enclosures and any additional wiring; cooling bodies and heat spreaders; thermal cladding, thermal potting material and thermal interface material; interwinding isolation material in inductor and transformer structures; dedicated thermal layers on printed circuit boards; PCB tracks and any enclosure(s) attached to the PCB structure that assist in thermal transport; capacitor housings; or any material that transports significant heat in a system.

To illustrate: consider a discrete $T O-220$ transistor package as shown in Figure 2.

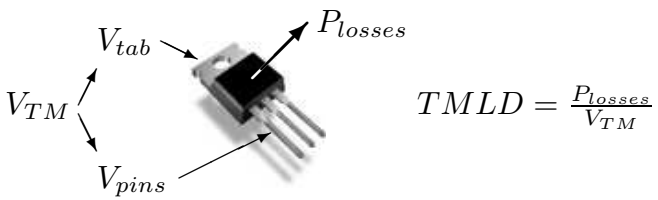

Fig. 2. TMLD determination for typical MOSFET in TO-220 package

The back plate and electrical pins have a combined volume of $211.18 \mathrm{~mm}^{3}$ that contributes towards conductive thermal management, this is then $V_{T M}$. Should the component inside the package develop $1 \mathrm{~W}$ of heat $\left(P_{\text {losses }}\right)$ due to its electrical operation, then it's TMLD would be $\frac{1}{211.18} \approx$ $4.7 \mathrm{~mW} / \mathrm{mm}^{3}$. Similar for a $T O-247$ package a TMLD value of $\approx 1.7 \mathrm{~mW} / \mathrm{mm}^{3}$ will result, indicating a reduced stress on thermal management. A few more component packages' TMLD values under the same conditions as above are shown in Table I for comparison.
TABLE I

THERMAL MANAGEMENT DENSITY (TMLD) VALUES FOR COMMERCIALLY AVAILABLE POWER MOSFET PACKAGES WITH $1 \mathrm{~W}$ OF ELECTRICAL LOSSES

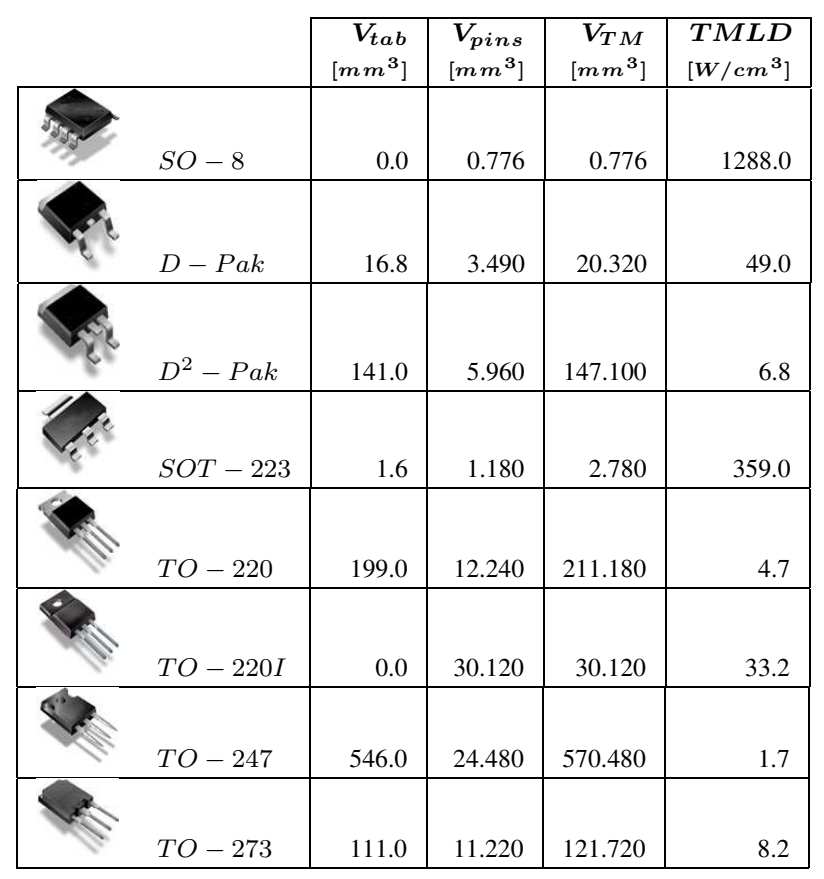

2) Thermal design rating: The optimal operation temperature of a component is a compromise between the lifetime-, reliability- and the level of electromagnetic excitation of a component: low operating temperatures improves reliability whereas higher temperatures offer more efficient material usage due to a higher electromagnetic excitation capability. The thermal design rating (TDR) quantifies the thermal performance of a component, or system, as a result of its applied thermal management. This according to a set of optimal operation temperatures which are characteristic to a set objective or goal, which may include but are not limited to reliability and power density. This rating system evaluates the thermal performance of a single component by how far it's operating temperature, either surface or internally measured, is removed from the prescribed optimum temperature. The quantitative rating is performed by means of a modified version of the statistical normal distribution curve, shown in Figure 3 and given by (1). The maximum material temperature, $T_{\max }$, is available in the literature whereas the optimal temperature is the designer's prerogative to adjust according to a set objective and could be empirical in nature.

Rating of more than one component and complete systems involves averaging the respective rating information for each individual component to arrive at a common figure of merit, or performance appraisal for the complete system. A weighted 


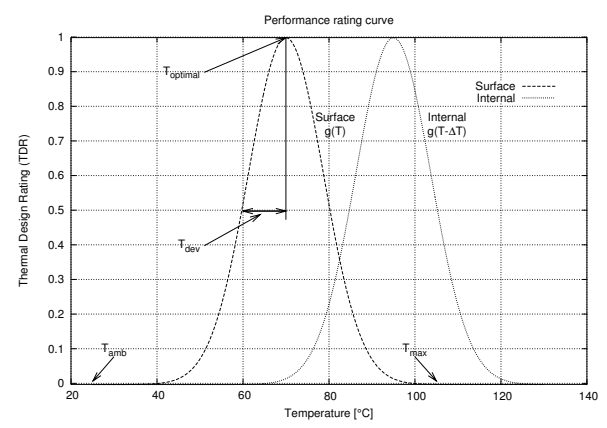

$$
\begin{aligned}
& T D R \equiv g(T)=\exp \left[-4\left(\frac{T-T_{\text {optimal }}}{T_{\text {max }}-T_{\text {optimal }}}\right)^{2}\right] \\
& T_{\text {dev }}=\frac{\left(T_{\text {max }}-T_{\text {optimal }}\right)}{4} \quad \text { (can be adjusted as appropriate) } \\
& T D R_{w s}=\frac{a_{1} T D R_{1}+a_{2} T D R_{2}+\ldots+a_{n} T D R_{n}}{n} \\
& T D R_{\text {band }}=\frac{n \mapsto(T D R>\alpha)}{n}
\end{aligned}
$$

Fig. 3. Typical thermal design rating curve

average of the individual components temperatures can be evaluated, with the weights adjusted to emphasise the role of crucial components, given by (2) for $(0 \leq a \leq 1)$.

The motivation for choosing a certain weighing function relies on the objective of the evaluation. One could, for example, have similar weights for common components: all capacitors together, all ferrites together and all resistors grouped together.

A second option to evaluate thermal design rating of systems is to calculate the ratio of how many components operate in a predefined optimal temperature band, defined as having a thermal performance rating of " $\alpha$ " or higher, against the total amount of components that are considered, as shown in (3). The " $\longmapsto$ " operator counts the number of elements for which the "argument", in this case temperature rating in optimal band, is true. A converter with all the power components operating at exactly its optimal value will have a $T D R_{W S}$ and $T D R_{\text {band }}$ of 1 , the asymptotic ideal value.

For example, consider an electrolytic filter capacitor and coupled inductor of a power electronic converter, as shown in Figure 5. Applying the thermal design rating per individual component using (1), for a power density objective as defined in Table II, the capacitors $T D R_{P D}$ can be seen to be a mere $0.2 \%$, whereas the coupled inductor has a $98.8 \% T D R_{P D}$.

TABLE II

THERMAL OBJECTIVE TEMPERATURE DEFINITIONS

\begin{tabular}{|l|c|c|c|c|}
\cline { 2 - 5 } \multicolumn{1}{c|}{} & \multicolumn{2}{c|}{ Power density } & \multicolumn{2}{c|}{ Reliability } \\
\cline { 2 - 5 } \multicolumn{1}{c|}{} & $C$ & $L$ & $C$ & $L$ \\
\hline$T_{\text {optimal }}$ & $70^{\circ} \mathrm{C}$ & $75^{\circ} \mathrm{C}$ & $50^{\circ} \mathrm{C}$ & $55^{\circ} \mathrm{C}$ \\
\hline$T_{\max }$ & $105^{\circ} \mathrm{C}$ & $130^{\circ} \mathrm{C}$ & $105^{\circ} \mathrm{C}$ & $130^{\circ} \mathrm{C}$ \\
\hline
\end{tabular}

Similarly applying a reliability objective, their $T D R_{r}$ are $51.2 \%$ and $81.4 \%$ respectively.

The implication of the performance indicators as outlined above will become clear in the concept illustration section to follow.

\section{CONCEPT ILLUSTRATION}

The concept will be illustrated from two different point of views, first from a technology platform and thereafter a thermal management point of view. This to show how the figures of merit still reflect the appropriate level of integration irrespective of the technology drivers involved.

Technology platform point of view: A dc-dc, 42/14V@10A converter for automotive applications in synchronous buck topology is implemented in three technology platforms using functional and packaging elements integration. Figure 4 shows the benchmark converter, constructed in conventional discrete technology (double sided PCB, wire-wound inductor and discrete heat sinking) and the novel concepts: Heat conductor converter, Lead frame converter and PCB embedded converter. Heat conductor converter is implemented on a thick film ceramic substrate and its salient feature is the multifunctional busbar that collects the heat from both active and passive components and delivers it to the heat sink, implements one turn of the inductor winding and provides mechanical support. Lead frame converter employs lead frame technology for implementing the whole converter, including the inductor which windings are manufactured as a part of the lead frame. Plastic composite nanocrystalline Vitroperm is employed to enhance the inductance. PCB converter utilises embedded passives technology (Ferrite Polymer Composite material C303) and heat spreading and thermal vias for enhanced thermal management.

TABLE III

INTEGRATION LEVEL COMPARISON AMONGST TECHNOLOGY PLATFORMS

\begin{tabular}{|c|c|c|c|c|}
\cline { 2 - 5 } \multicolumn{1}{c|}{} & $\begin{array}{c}\text { Discrete } \\
\text { converter }\end{array}$ & $\begin{array}{c}\text { Heat } \\
\text { conductor } \\
\text { converter }\end{array}$ & $\begin{array}{c}\text { Lead } \\
\text { frame } \\
\text { converter }\end{array}$ & $\begin{array}{c}\text { PCB } \\
\text { embedded } \\
\text { converter }\end{array}$ \\
\hline$K_{I}[-]$ & 1.00 & 1.17 & 1.4 & 2.00 \\
\hline$K_{P}[-]$ & 1.15 & 1.53 & 2.0 & 1.00 \\
\hline$\eta[W / l]$ & 591.9 & 4613 & 4626 & 5083 \\
\hline
\end{tabular}

Table III shows the values of integration levels of the three demonstrators and a discrete benchmark converter. The values of power densities are also given. PCB embedded converter has 


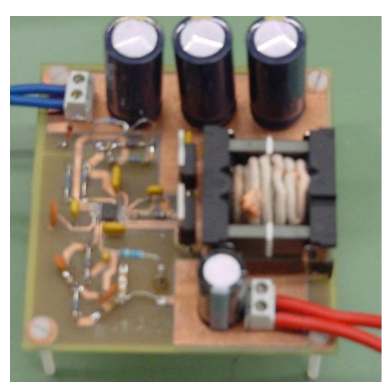

(a) Discrete benchmark converter

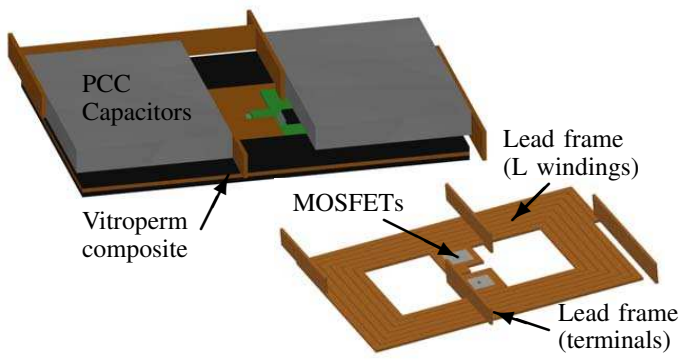

(c) Lead frame converter

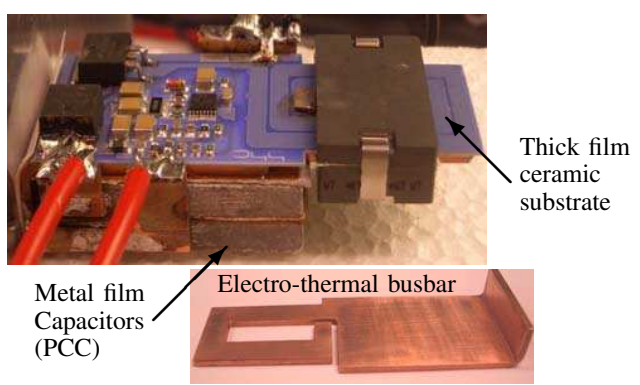

(b) Heat conductor converter

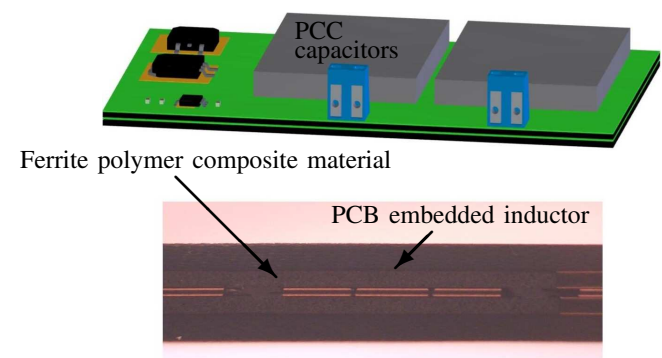

(d) PCB embedded converter

Fig. 4. Concept illustration dc-dc electronic power converters built on different technology platforms

the highest value of the functional elements integration due to the integrated inductor, Lead frame converter has the highest level of packaging elements integration due to its simple construction and few construction parts. All three converters have power densities an order of magnitude higher than the discrete benchmark converter.

Thermal management point of view: An illustration outlining the above thermal management figure of merit concept, is applied to an "off-the-line" ac-dc converter delivering 1A@5V and 1.25A@12V in discrete and higher integration level implementations. The figure of merit for thermal effectiveness is used to quantify the thermal performance of the 20W "off-the-line" Flyback ac-dc converter, as well as the performance improvement of its thermally redesigned counterpart, shown in Figure $5 \& 6$ respectively [8].

Figure 5(a) shows the benchmark converter, constructed in conventional discrete technology (single sided PCB, wire-wound coupled inductor and discrete heat sinking) delivering 1A@5V and 1.25A@12V, addressing increased thermal management by functional and packaging elements integration of mainly the PCB. The improved converter realisation is shown in Figure 6(a). It utilises the same discrete technology as the benchmark version except for the use of flexible PCB technology as advanced packaging element and the removal of discrete heatsinking due to functional integration of the heatsink in the PCB, enhanced by thermal vias and inter component thermal interface material. The categories and their respective parameter values, for achieving high power density and achieving prolonged reliability of converters, used in this case study is
TABLE IV

THERMAL OBJECTIVE TEMPERATURE DEFINITIONS PER COMPONENT CATEGORY

\begin{tabular}{|l|c|c|c|}
\cline { 2 - 3 } \multicolumn{1}{c|}{} & \multicolumn{2}{c|}{$T_{\text {optimal }}\left[{ }^{\circ} \mathrm{C}\right]$} & \multirow{2}{*}{$T_{\max }\left[{ }^{\circ} \mathrm{C}\right]$} \\
\cline { 2 - 3 } \multicolumn{1}{c|}{} & Power density & Reliability & \\
\hline Capacitors & 70 & 50 & 105 \\
\hline Magnetic components & 75 & 55 & 130 \\
\hline Semiconductors & 110 & 75 & 150 \\
\hline Resistors & 55 & 35 & 80 \\
\hline
\end{tabular}

given in Table IV.

Table V shows the values of the respective performance indicators as determined for the benchmark and improved converters. From this table it can be seen that the functional element integration level has increased incrementally from 1.00 to 1.29 due to the PCB and electrolytic capacitor now also acting as heat spreader and exchanger, performing as thermal functional element. This illustrates the $K_{I}$ improvement method using packaging elements as functional elements, as described in Section II-A.1. This improvement is not that significant since there is not much functional elements integration (all the components are still in the discrete form). For this level of functional element integration to improve significantly in future versions of this converter, the integration of passive functional elements into the PCB are considered.

The packaging elements integration level has been improved from 1.55 to 2.06 by applying both the multifunctional packaging elements and sharing packaging elements methods, as 


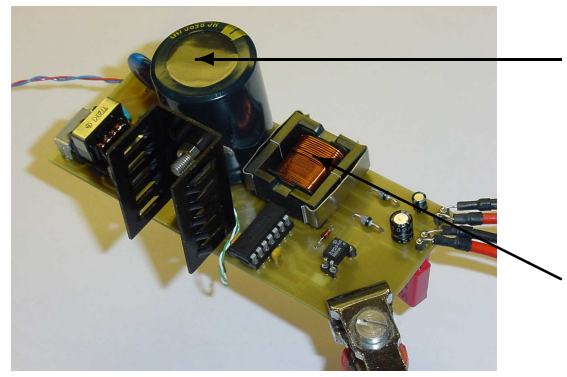

(a) ac-dc converter

\begin{tabular}{|c|c|}
\hline Temp & $27.5^{\circ} \mathrm{C}$ \\
\hline$T D R_{P D}$ & $0.2 \%$ \\
\hline$T D R_{r}$ & $51.2 \%$ \\
\hline Temp & $72^{\circ} \mathrm{C}$ \\
\hline$T D R_{P D}$ & $98.8 \%$ \\
\hline$T D R_{r}$ & $81.4 \%$ \\
\hline
\end{tabular}

(b) TDR values

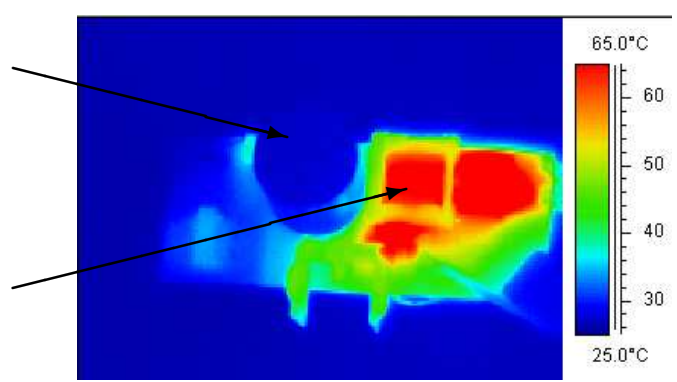

(c) thermal measurement

Fig. 5. Applying the thermal design rating method

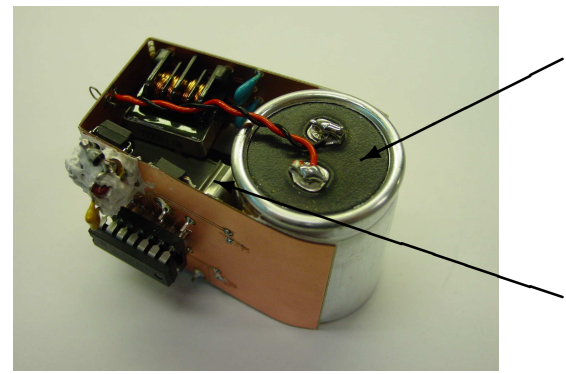

(a) improved ac-dc converter

\begin{tabular}{|c|c|}
\hline Temp & $40.3^{\circ} \mathrm{C}$ \\
\hline$T D R_{P D}$ & $5.6 \%$ \\
\hline$T D R_{r}$ & $88.3 \%$ \\
\hline \multicolumn{2}{|c|}{} \\
\hline$T e m p$ & $51.4^{\circ} \mathrm{C}$ \\
\hline$T D R_{P D}$ & $47.8 \%$ \\
\hline$T D R_{r}$ & $99.1 \%$ \\
\hline
\end{tabular}

(b) TDR values

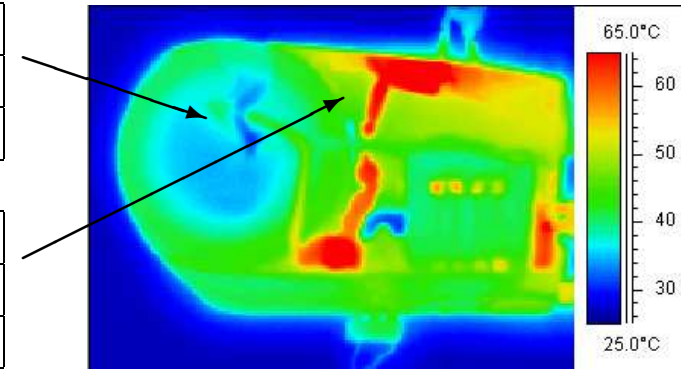

(c) thermal measurement

Fig. 6. Concept illustration ac-dc electronic power converters

TABLE V

PERFORMANCE INDICATOR COMPARISON

\begin{tabular}{|c|l|l|r|r|}
\hline \multicolumn{2}{|c|}{ Parameter } & Description & Benchmark & Improved \\
\hline \hline \multicolumn{2}{|c|}{$K_{I}$} & Functional element integration level [-] & 1.00 & 1.29 \\
\hline \multicolumn{2}{|c|}{$K_{P}$} & Packaging element integration level [-] & 1.55 & 2.06 \\
\hline \multicolumn{2}{|c|}{ TMLD } & Thermal management loss density $\left[\mu \mathrm{W} / \mathrm{mm}^{3}\right]$ & 95.85 & 813.89 \\
\hline $\begin{array}{c}\text { Power density } \\
\text { TDR }\end{array}$ & $w s(a=1)$ & Thermal design rating - power density objective (Weighted sum) [\%] & 18.5 & 11.4 \\
\cline { 2 - 6 } & $\alpha=1 \%$ & Thermal design rating - power density objective (Optimal band > 1\%) [\%] & 29.0 & 53.1 \\
\hline \multirow{3}{*}{$\begin{array}{c}\text { Reliability } \\
\text { TDR }\end{array}$} & $w s(a=1)$ & Thermal design rating - reliability objective (Weighted sum) [\%] & 49.3 & 80.2 \\
\cline { 2 - 6 } & $\alpha=1 \%$ & Thermal design rating - reliability objective (Optimal band $>1 \%)[\%]$ & 96.8 & 100.0 \\
\cline { 2 - 6 } & $\alpha=50 \%$ & Thermal design rating - reliability objective (Optimal band $>50 \%)[\%]$ & 48.4 & 96.9 \\
\hline \multicolumn{2}{|c|}{$\eta$} & Thermal design rating - reliability objective (Optimal band > 85\%) [\%] & 3.2 & 46.9 \\
\hline \multicolumn{2}{|c|}{$\eta$} & Power density [W/l] & 150 & 300 \\
\hline
\end{tabular}

described in Section II-A.2, to mainly the PCB and electrolytic capacitor. The PCB now performs electrical interconnection, provides insulation, protection and mechanical support as well as aids in thermal management, whereas the electrolytic capacitor now also provides mechanical support.

These integration level indicators also reflect on the overall packaging effectiveness of the system, defined as

$$
\eta_{v}=\frac{V_{F E}}{V_{T O T}}
$$

where $V_{F E}$ is the volume occupied by all functional elements as defined in Section II, and $V_{T O T}$ is the total converter volume.

The improved converter has a packaging effectiveness of more than double that of the benchmark model. This is mostly due to the more efficient three dimensional spatial layout of the components, which resulted in the reduction of the converter volume, while the total functional elements volume remained approximately the same. 
Important to note is that, although thermal management improvement was the technology driver in this case, the functional and packaging element integration level increased accordingly, indicating the close relationship between the integration, packaging and thermal management. The figures of merit are therefore able to indicate these integrally linked relationships.

Furthermore, Table $\mathrm{V}$ shows that the benchmark thermal management exhibits $\mathrm{a} \approx 96 \mu \mathrm{W} / \mathrm{mm}^{3}$ thermal loss density in its thermal conducting material; whereas the improved design exhibits a density of more than eight times this value, $\approx 814 \mu \mathrm{W} / \mathrm{mm}^{3}$. This indicates a much more intensified use of the invested material in the improved converters thermal design and can be traced back in the power densities achieved of twice that of the benchmark design.

Table V further shows the TDR values calculated for the whole system using both "weighted sum"(ws) and "optimal band" $(\alpha)$ averaging methods. The thermal design rating values (TDR) for the overall converter where calculated using a weighted sum method (ws) with all weights being equal to 1, i.e. $a_{1}=a_{2}=\ldots=a_{n}=1$ and secondly by setting an optimal band at $1 \%, 50 \%$ and $85 \%$ of the theoretical optimum of the overall converter respectively ( $\alpha=0.01 ; 0.5 ; 0.85$ ). When comparing the thermal design ratings for the overall converters based on power density objective the ratings show that the thermal management of the improved design has improved to such an extent that $53.1 \%$ of the components now operate in the set optimal thermal band of $1 \%$, instead of the $29 \%$ of the components in the conventional converter. $0 \%$ of the components operate in the set optimal bands of $50 \%$ and $85 \%$. This shows the huge margin of improvement that still exists for this converter in this area.

The thermal design rating for reliability gives a whole different perspective on the improved design. Almost all components operate in an optimal band of 50\% and as much as $46.9 \%$ operate in the optimal band of $85 \%$ in comparison with nearly half the components of the conventional design operating in the $50 \%$ optimal band and only $3.2 \%$ operating in the $85 \%$ optimal band.

The thermal management figures of merit have enabled the performance appraisal of a conventional Flyback converter as well as the improvement comparison of its thermally redesigned counterpart. The intensity in which the thermal management material in the converter is being utilised has also been quantified and allows direct comparison with other systems, or designs. The figures of merit therefore can be seen to form an integral part of the optimisation cycle when designing for thermal management in converters. In general, it can therefore be seen that applying the figures of merit make a quantitative comparison possible to comment on the integration level and thermal management performance of any power electronic converter system.

\section{CONCLUSION}

A giant leap forward for converters in the power electronic industry is possible by increasing the level of integration. By incorporating multi-functional parts and components, instead of conventional discrete component technology, a converter can be put together using fewer parts and less manufacturing processes. This immediately reduces size and cost and makes power electronics viable to meet the low cost and high performance demands set by demanding applications. The implementation of various methods and technologies in achieving the higher integration level can lead to many functional solutions. It remains difficult to identify which solution is the best. For this figures of merit are required covering not only electrical design parameters, but packaging, integration and thermal management as well. This to aid in the systematic design of power electronic converters with high integration levels. Figures of merit to meet this demand has been discussed and applied to a case study herein, indicating how it is possible to obtain an objective look on the overall design of integrated power electronic converters and their performance. If these figures of merit are adopted in converter design one can systematically design power electronics with higher integration levels resulting in cheaper, more power dense converters aiding the power electronic industry to meet the low cost and high performance demand.

\section{REFERENCES}

[1] J. Van Wyk, "Power electronics technology at the dawn of a new centurypast achievements and future expectations," in Conf. proc. of Power Electronics and Motion Control Conference, vol. 1, August 2000, pp. 9-20.

[2] F. Lee and P. Barbosa, "The state-of-the-art power electronics technologies and future trends," in Conf. Proc. of Transmission and Distribution Conference and Exposition, vol. 2, October 2001, pp. 1188-1193.

[3] N. P. Suh, The principles of design. New York: Oxford University Press, 1990.

[4] S. Shina, Concurrent Engineering and Design for Manufacture of Electronics Products. New York: Van Nostrand Reinhold, 1991.

[5] S. Mathuna, P. Byrne, G. Duffy, W. Chen, M. Ludwig, T. O’Donnell, P. McCloskey, and M. Duffy, "Packaging and integration technologies for future high-frequency power supplies," IEEE Transactions on Industrial Electronics, vol. 51, no. 6, pp. 1305-1312, December 2004.

[6] E. Waffenschmidt and J. Ferreira, "Embedded passives integrated circuits for power converters," in conf. proc. of Power Electronics Specialists Conference, vol. 1. IEEE, June 2002, pp. 12 - 17 .

[7] F. Lee, J. van Wyk, D. Boroyevich, G.-Q. Lu, Z. Liang, and P. Barbosa, "Technology trends toward a system-in-a-module in power electronics," IEEE Circuits and Systems Magazine, vol. 2, no. 4, pp. 4-22, Fourth Quarter 2002.

[8] E. de Jong, J. Ferreira, and P. Bauer, "Improving the thermal management of AC-DC converters using integration technologies," in Conf. Proc. of Industry Applications Conference, vol. 4. IEEE, October 2004, pp. 2315-2322.

[9] J. Popović and J. Ferreira, "An approach to deal with packaging in power electronics," IEEE Transactions on Power Electronics, vol. 20, no. 3, pp. 550-557, May 2005.

[10] H. Iwamoto, E. Motto, J. Achhammer, M. Iwasaki, M. Seo, and T. Iwagami, "New intelligent power modules for appliance motor control," in Conf. Proc. of Applied Power Electronics Conference and Exposition, vol. 2, 2001, pp. 1051-1056.

[11] E. de Jong, J. Ferreira, and P. Bauer, "Evaluating Thermal Management Efficiency in Converters," in Conf. Proc. of Power Electronic Specialist Conference. IEEE, June 2004, pp. 4881-4887. 